\title{
Revascularização do miocárdio minimamente invasiva
}

\author{
Carlos A. TELES*, Ênio BUFFOLO* Antônia PETRIZZO** Expedito RIBEIRO**, \\ Lélio A. SILVA ${ }^{\star *}$, Wilson MATHIAS Jr. ${ }^{\star \star}$
}

RBCCV 44205-294

Teles C A, Buffolo E, Petrizzo A, Ribeiro E, Silva L A, Mathias Jr. W - Revascularização do miocárdio minimamente invasiva. Rev Bras Cir Cardiovasc 1996; 11 (2): 82-5.

RESUMO: Objetivo: Tendência atual em todas as áreas da cirurgia aponta para táticas e técnicas cirúrgicas que tornem o ato operatório cada vez menos invasivo. O objetivo do presente estudo foi avaliar a viabilidade da revascularização do miocárdio através de minima incisão torácica (minitoracotomia esquerda), sem circulaçāo extracorpórea.

Casuística e Métodos: De setembro de 1995 a março de 1996, um total de 19 pacientes portadores de lesōes isoladas do ramo interventricular anterior (RIA) e/ou diagonal (Dg) foram submetidos a revascularizaçāo do miocárdio, tendo, como via de acesso, toracotomia anterior paraesternal esquerda, de aproximadamente $7 \mathrm{~cm}$ de extensão, ao nivel do $4^{2}$ espaço intercostal. Através dessa incisão foi dissecada a artéria torácica interna esquerda (ATIE) em extensāo suficiente para a realizaçăo da anastomose com o RIA efetuada sem circulação extracorpórea. No $2^{\circ}$ dia de pós-operatório esses pacientes foram submetidos a arteriografia e ecoDoppler transtorácico da ATIE para verificação das condiçōes do enxerto e da persistência de fluxo pelos ramos intercostais não ligados por ocasião da dissecção parcial da artéria.

Resultados: Todos os pacientes tiveram boa evolução pós-operatória. O resultado da ATIE realizado em 16 dos 19 pacientes operados mostrou padrōes angiográficos excelentes em 13 deles. Não se demonstrou contrastação dos ramos intercostais não ligados durante a dissecção. O Doppler transtorácico se correlacionou com as condiçōes dos enxertos.

Conclusão: Os resultados iniciais obtidos na revascularização do miocárdio através de minitoracotomia esquerda sugerem ser esta uma boa alternativa tática para portadores de lesão isolada do RIA com excelente resultado estético.

DESCRITORES: Revascularização do miocárdio, métodos. Cirurgia minimamente invasiva, métodos. Artérias mamárias, cirurgia. Vasos coronários, cirurgia. Revascularização do miocárdio, cirurgia.

\section{INTRODUÇÃO}

Desde as primeiras pontes aorto-coronárias realizadas na década de 60 , a cirurgia cardíaca evoluiu consideravelmente em suas técnicas e indicaçōes. Com isso, um número cada vez maior de pacientes tem sido beneficiado nos últimos anos.

Mais recentemente, vem sendo notada preocupação crescente na cirurgia cardiaca com a simpli- ficação do ato operatório, a exemplo do que ocorre com todas as demais áreas da cirurgia. Técnicas menos invasivas, não raro, implicam em custos menores e diminuição da mortalidade e da morbidade. Importante passo nesse sentido foi dado pela introdução das técnicas de revascularização do miocárdio sem circulação extracorpórea.

Contudo, até recentemente poucas alternativas haviam sido propostas no sentido de simplificar as

Trabalho realizado na Escola Paulista de Medicina - UNIFESP e no Hospital Unicór, Săo Paulo, SP, Brasil.

Apresentado ao $23^{\circ}$ Congresso Nacional de Cirurgia Cardiaca, Recife, PE, 20 a 23 de março, 1996.

* Da Escola Paulista de Medicina.

** Do Hospital Unicór.

Endereço para correspondência: Carlos A. Teles. Rua Borges Lagoa, 783. $5^{2}$ andar. CEP: 04038-032 São Paulo, SP, Brasil. 
Teles C A, Buffolo E, Petrizzo A, Ribeiro E, Silva L A, Mathias Jr. W - Revascularização do miocárdio minimamente invasiva. Rev Bras Cir Cardiovasc 1996; 11 (2): 82-5.

vias de acesso, substituindo as grandes incisões clássicas (esternotomias) por incisões menores, menos agressivas.

O objetivo do presente estudo foi avaliar a viabilidade da realização da revascularização do miocárdio através de mínima incisão torácica (minitoracotomia esquerda), sem circulação extracorpórea, conforme proposto inicialmente por BENETTI, em $1994^{*}$, e CALAFIORE et al. em 1995 *.

\section{CASUISTICA E MÉTODOS}

No período de setembro de 1995 a março de 1996 , um total de 19 pacientes portadores de insuficiência coronária crônica foram submetidos a revascularização do miocárdio através de mínima incisão paraesternal esquerda, sem circulaçāo extracorpórea.

Destes, $13(68,4 \%)$ eram do sexo masculino e $6(31,6 \%)$ do sexo feminino, com idades variando de 39 a 68 anos (média de 51 anos). Todos os pacientes foram operados em caráter eletivo.

O critério para seleção dos pacientes foi insuficiência coronária crônica caracterizada por lesão isolada do ramo interventricular anterior (RIA) e/ou diagonal $(\mathrm{Dg})$, com indicação de tratamento intervencionista (cirurgia ou angioplastia). Em 2 pacientes, além da anastomose da ATIE com a RIA, foi realizada outra ponte para a diagonal, utilizando a artéria radial como enxerto composto.

\section{TÉCNICA OPERATÓRIA}

Após monitoração do ritmo cardíaco, pressão arterial, pressão venosa central e débito urinário através de eletrodos e cateteres, foi feita indução anestésica com neuroleptoanalgesia clássica e intubação orotraqueal. Em decúbito lateral realizou-se a punção do espaço peridural com injeção de $5 \mathrm{mg}$ de morfina visando prolongar a analgesia no período pós-operatório.

Com o paciente em semidecúbito lateral direito, procedeu-se à realização de toracotomia anterior paraesternal esquerda por planos, ao nivel do sulco inframamário nas mulheres e inframamilar nos homens, com aproximadamente $7 \mathrm{~cm}$ de extensão; abertura da cavidade torácica ao nível do $4^{\circ}$ espaço intercostal. Com o auxílio de afastador auto-estático, procedeu-se à desinserção da cartilagem costal superior, embora em poucos casos tenha sido necessária sua ressecção.

\footnotetext{
* Comunicação pessoal.
}

Após identificação, iniciou-se a dissecção cuidadosa da ATIE junto ao ângulo medial da incisão, sob visão direta, proximal e distalmente, em extensão suficiente para a realização da anastomose com o RIA. Os ramos intercostais que foram se tornando visiveis e abordáveis foram ligados.

Com o afastamento lateral do pulmão, o pericárdio foi identificado, incisado longitudinalmente e levemente tracionado para melhor exposição da artéria coronária.

Identificada a artéria a ser abordada (RIA), foi administrada heparina na dose de $2 \mathrm{mg} / \mathrm{kg}$ de peso e seccionada distalmente a (ATIE). A técnica empregada na realização da anastomose sem circulação extracorpórea foi a mesma já extensivamente descrita em relatos anteriores $(1,2)$.

Completada a anastomose, procedeu-se à neutralização da heparina com sulfato de protamina $e$ à revisão da hemostasia. A drenagem da cavidade pleural precedeu o fechamento da parede torácica por planos.

No $2^{\circ}$ dia de pós-operatório os pacientes foram submetidos a exame contrastado e eco-Doppler transtorácico da ATIE para verificação das condiçōes do enxerto e da anastomose e da persistência de fluxo pelos ramos intercostais não ligados por ocasião da dissecção parcial da artéria.

\section{RESULTADOS}

$\mathrm{O}$ ato operatório transcorreu sem anormalidades em todos os casos. Todos os pacientes tiveram boa evolução pós-operatória, com alta da UTI no $1^{\circ}$ dia de pós-operatório.

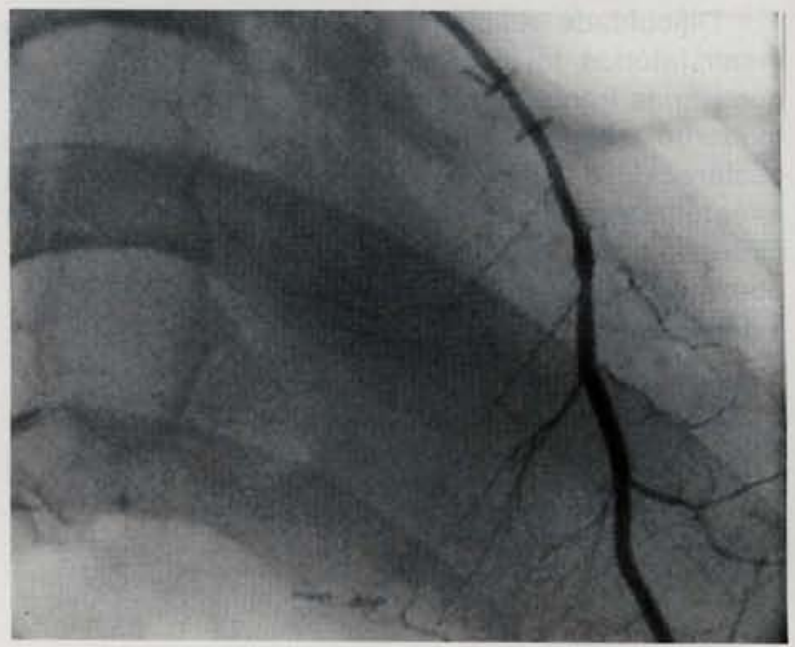

Fig. 1 - Angiografia de controle pós-operatório, contrastando a artéria torảcica interna esquerda (ATIE) e ramo interventricular anterior (RIA) 
Teles C A, Buffolo E, Petrizzo A, Ribeiro E, Silva L A, Mathias Jr. W - Revascularização do miocárdio minimamente invasiva. Rev Bras Cir Cardiovasc 1996; 11 (2): 82-5.

$O$ reestudo da ATIE foi realizado em 16 dos 19 pacientes operados e mostrou padrões angiográficos excelentes em 13 deles (Figura 1 ) (em 3 casos houve necessidade de reoperação, que foi realizada por esternotomia).

Em nenhum caso houve contrastação dos ramos intercostais não ligados durante a dissecção. O eco-Doppler transtorácico se correlacionou com o bom fluxo dos enxertos em todos os casos, com padrão de fluxo diastólico contrastando com o padrão de fluxo sistólico na artéria torácica interna direita controle (Figuras 2 e 3 ).

\section{COMENTÁRIOS}

A minitoracotomia esquerda constitui uma nova opção tática para a revascularização do miocárdio de pacientes portadores de lesões isoladas da RIA e/ou Dg.

Além das vantagens inerentes à não utilização da circulação extracorpórea já amplamente divulgadas em publicações anteriores $(1,2)$ (menor necessidade de transfusões de sangue, menor custo pela não utilização do circuito extracorpóreo e menor tempo de hospitalização etc), acrescente-se a recuperação pós-operatória mais rápida e resultado estético superior ao da incisão convencional.

Em nenhum dos casos operados houve alterações hemodinâmicas ou arritmias durante a realização das anastomoses.

A avaliação dos resultados com ecocardiografia Doppler transtorácica mostrou-se bastante eficiente quando correlacionada com os achados angiográficos e diante do método não invasivo, confirmando citações na literatura a respeito do assunto $(3,4)$.

Dificuldade adicional nos casos de resultados insatisfatórios foi representada pela ocorrência de coronárias intramiocárdicas e de calibre reduzido. É evidente que o paciente deve estar previamente esclarecido e a equipe cirúrgica preparada para a eventual necessidade de mudança do planejamento cirúrgico frente aos achados ou ocorrências no intraoperatório, situações em que se deve optar por esternotomia, para maior segurança.

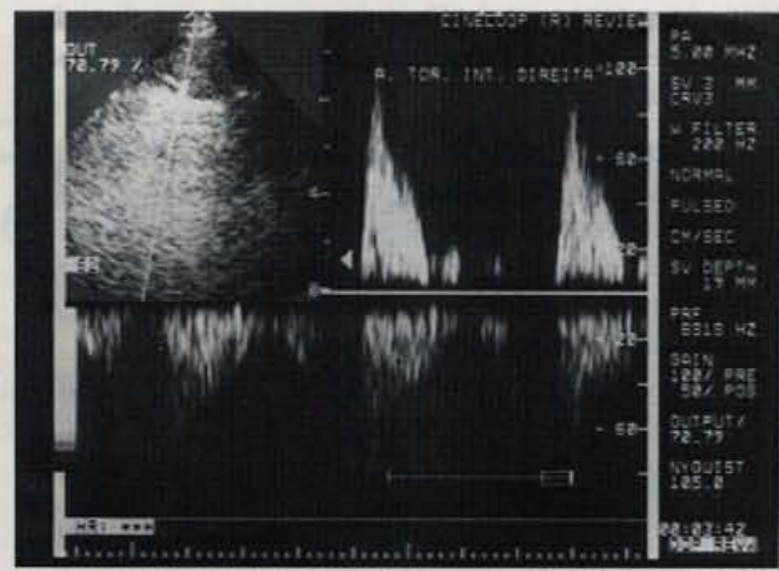

Fig. 2 - Eco-Doppler da a. torácica interna direita in situ. Fluxo bifásico com velocidade máxima ocorrendo na sistole.

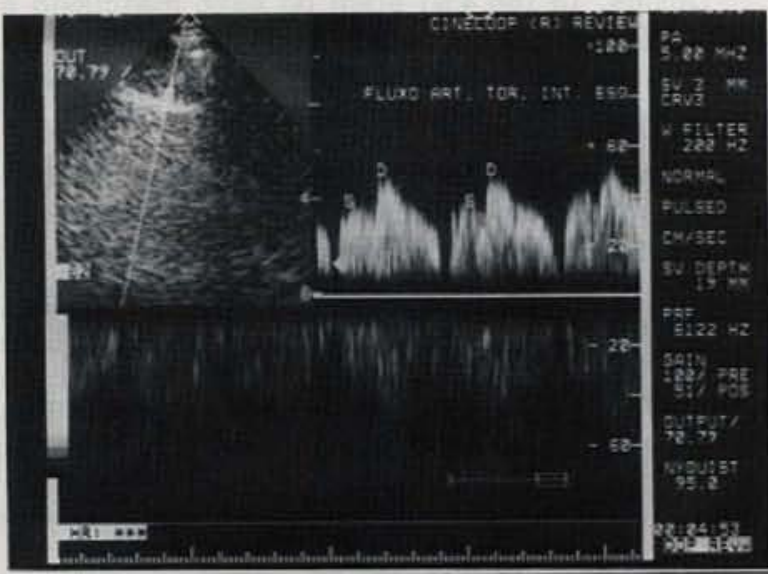

Fig. 3 - Eco-Doppler de enxerto de a. torácica interna direita patente. Fluxo bifásico com velocidade máxima ocorrendo na diástole.

Esta nova opção tática não necessita de dispositivos ou instrumentos especiais para sua execução, podendo ser realizada em qualquer Serviço de cirurgia cardiaca equipado para operações convencionais. Entretanto, é necessário sólida experiência da equipe cirúrgica, que deve estar bem familiarizada com operaçōes de revascularização do miocárdio sem circulação extracorpórea. 
Teles C A, Buffolo E, Petrizzo A, Ribeiro E, Silva L A, Mathias Jr. W - Revascularização do miocárdio minimamente invasiva. Rev Bras Cir Cardiovasc 1996; 11 (2): 82-5.

RBCCV 44205-294

Teles C A, Buffolo E, Petrizzo A, Ribeiro E, Silva L A, Mathias Jr. W - Myocardial revascularization through a minimum left thoracotomy approach. Rev Bras Cir Cardiovasc. 1996;11 (2): 82-5.

ABSTRACT: There is a tendency in all fields of surgery to move towards less invasive surgical techniques in recent years. The end point of this study was to analyse the possibility of myocardial revascularization through a minimum left thoracotomy approach. From September 1995 till March 1996 we operated on 19 patients that had isolated lesion of interventricular anterior artery (IAA) and/or diagonal. The anastomoses were made by a left minor thoracotomy, opening the pleura at the $4^{2}$ intercostal space. Through this incision we dissected the thoracic internal artery with ligation of only few branches. The anastomoses were performed without extracorporeal circulation, with a simple interruption of coronary blood flow. At the second postoperative day we studied the patients with angiography and transthoracic echo-Doppler, to verify patency and flow. All patients had uneventful recovery without major complications. Angiography was made in 16 out of 19 and showed patency in 13. The 3 patients with graft occlusion were reoperated on without complications. The presented technique did not involve ligation of the intercostal branches, however these branches were not demonstrated by angiography. Transthoracic Doppler showed good relationship with angiographic findings. The initial results with this technique suggest that we can have another option of minimum invasive myocardial revascularization for selected patients.

DESCRIPTORS: Myocardial revascularization, methods. Surgery minimally invasive, methods. Mammary arteries, surgery. Coronary vessels, surgery. Myocardial revascularization surgery.

\section{REFERÊNCIAS BIBLIOGRÁFICAS}

1 Buffolo E, Andrade J C S, Succi, J E et al. - Direct myocardial revascularization without extracorporeal circulation: technique and initial results. Texas Heart Inst J 1985; 12: 33-41.

2 Buffolo E, Andrade J C S, Branco J N R, Aguiar L A, Ribeiro E E, Jatene A D - Myocardial revascularization without extracorporeal circulation. Eur J Cardio-thorac Surg 1990; 4: 504-8.
3 Crowley J J \& Shapiro L M - Noninvasive assessment of left internal mammary artery graft patency using transthoracic echocardiography. Circulation 1995; 92: 25-30.

4 Kyo S, Matsumura N, Yokote Y, Takamoto S, Omoto R - Evaluation of patency of internal mammary artery grafts: a comparison of two-dimensional Doppler echocardiography and coronary angiography. $J$ Cardiol 1990; 20: 606-16. 